\title{
Philosophiques
}

\section{"Le philosophe chat ou les ruses du désir », de Roger Savoie}

\section{Marc Chabot}

Volume 8, numéro 1, avril 1981

URI : https://id.erudit.org/iderudit/203157ar

DOI : https://doi.org/10.7202/203157ar

Aller au sommaire du numéro

Éditeur(s)

Société de philosophie du Québec

ISSN

0316-2923 (imprimé)

1492-1391 (numérique)

Découvrir la revue

Citer ce document

Chabot, M. (1981). « Le philosophe chat ou les ruses du désir », de Roger Savoie. Philosophiques, 8(1), 185-196. https://doi.org/10.7202/203157ar d'utilisation que vous pouvez consulter en ligne.

https://apropos.erudit.org/fr/usagers/politique-dutilisation/ 


\title{
«LE PHILOSOPHE CHAT \\ OU LES RUSES DU DÉSIR», DE ROGER SAVOIE
}

\author{
par Marc Chabot
}

Le livre publié à l'été par Roger Savoie: Le philosopbe cbat ou les ruses $d u$ désir semblerait être de ceux qui ne se commentent pas ou qui prennent place dans une culture sans avoir d'intention ou de volonté quelconque. C'est le genre de livre qui vous glisse entre les mains aussitôt qu'on voudrait saisir une thèse centrale ou simplement une idée qui dominerait l'oeuvre. Mais il s'agit là d'une concession trop grande faite à un objet culturel. On n'écrit pas pour rien, on ne publie pas pour rien non plus, et on ne vit pas pour rien.

Pour ma part, je tiens à spécifier que je n'ai nullement l'intention de pourfendre Le philosopbe chat, ni d'en faire une critique élogieuse. Ces deux attitudes sont néfastes et surtout stériles. Il est toutefois possible de lire un livre tout en se conservant en santé, tout en restant soi-même, sans que cette attitude ne se dévoile comme fausse résistance.

Les quelques commentaires que je formulerai sont écrits sous le mode épistolaire. Ce choix s'est imposé à moi en lisant les dernières pages du livre de Roger Savoie:

Je ne crois pas en l'écriture miroir, qui est proprement une écriture spéculative, une écriture spéculaire. Il y a des écritures qui s'arrachent les cheveux pour savoir si la parole accouchée ressemble à son géniteur, ou s'ils ont réussi à communiquer ce qu'ils voulaient bien dire. J'avoue que j'ai commencé ces pages sans savoir où $j$ 'allais, ni ce que je voulais. Une chose certaine: je n'avais pas du tout la préoccupation de transmettre un message qui aurait été l'image approchante de ma pensée ${ }^{1}$.

1. Roger Savoie, Le philosophe chat ou les ruses du désir, Quinze/prose exacte, Montréal, 1980, p. 163. 
C'est une lettre que j'écris à ce philosophe Roger Savoie, ou à ce qui en reste. C'est une lettre que j'écris à ce personnage multiple qu'est aussi l'anti-philosophe Roger Savoie. Je m'adresse à tous ses «je» et à tous ses «moi». Je finirai bien, je suppose, par m'approcher de quelqu'un, peu m'importe que ce soit la bête raisonnante ou le fou de désir!

\title{
Cher Roger,
}

Pas une minute je n'ai cessé de penser à Kant en lisant $L e$ philosophe chat. Kant, ce fou de la raison, cet obsédé de la mise en boîte, ce manipulateur de concepts, celui qui, dit-on, a su provoquer dans la discipline philosophique la révolution qu'avaient connue les sciences physiques avec Copernic. Or ce philosophe écrivait:

\begin{abstract}
. chez beaucoup de gens, et chez ceux-là surtout qui ont la plus grande expérience de l'usage de la raison, il se produit, s'ils sont assez sincères pour l'avouer, un certain degré de misologie, je veux dire de haine de la raison. Car, après avoir fait le compte de tous les avantages qu'ils peuvent retirer (...), ils arrivent à conclure que réellement ils ont eu plus de peine que de bonheur, c'est pourquoi en considérant la catégorie d'hommes inférieurs qui se laissent diriger davantage par l'instinct naturel, et qui n'accordent à leur raison que peu d'empire sur leur conduite, ils éprouvent, à leur égard, plus d'envie que de mépris ${ }^{2}$.
\end{abstract}

Cette souffrance nommée "misologie», je crois qu'elle nous habite encore et peut-être davantage que du temps de Kant. Il faut être philosophe pour la sentir, car le désir qui ne cesse de nous glisser entre les doigts, le corps que nous n'arrivons jamais à penser en entier font des philosophes des êtres déçus de l'impossibilité de tout dire et de tout raisonner.

Ton livre suppose d'ailleurs cette première déception philosophique: devant l'impossibilité de philosopher sur le corps, il faut se laisser aller dans notre corps, lui dire un beau gros OUI et le vivre, même si pour cela, il faut abandonner l'idée de comprendre et de donner du sens à nos actes.

Nous autres philosophes nous sommes souvent rudement mis à l'épreuve par notre objet de travail: la pensée. Théoriquement tout est pensable, tout est organisable. Les alternatives sont

2. E. Kant, Fondements de la métaphysique des moeurs, Traduction Hatier, Paris, 1963, p. 19. 
nombreuses, multiples et elles remplissent nos cerveaux rêveurs. D'un monde rationnel et froid à un monde de désir fou et flou, il n'y a qu'un pas. En te donnant comme objectif d'aller jusqu'au bout de l'incroyance, tu nous rends, sans le vouloir, un fier service. En nous rappelant que dans l'histoire occidentale le désir a été assassiné, tu nous aides à vivre au quotidien. Les philosophes, il ne faut pas l'oublier, même quand ils deviennent des anti-philosophes, n'en continuent pas moins de produire des pensées. Évidemment, ton intention n'est pas de "produire». C'est une liberté que je te concède avec facilité. Mais laisseras-tu aux lecteurs le soin de faire ce qu'ils veulent de ton livre? Probablement que cela ne te préoccupe nullement, puisque ce livre est écrit tout à fait gratuitement et sans intention.

Mais avant de parler des "ruses du désir», je voudrais m'arrêter quelques instants sur ces intentions inexistantes. Il peut sembler qu'il s'agit là d'un problème grave d'avoir des intentions. $\mathrm{Tu}$ laisses entendre que rien n'est prévisible, que nous nous trompons chaque fois que nous essayons de comprendre. Il n'y aurait rien à penser, rien à saisir, rien à déterminer, rien à dire même.

Pourquoi écrire, sinon pour le plaisir? Même celui de voir jusqu'où ça peut aller. Ce n'est pas un jeu de mots, c'est un risque que je prends, car il se pourrait bien que ce plaisir que j'avais se mue en souffrance, en peine, en pleurs. Peut-être y trouverai-je tout à fait autre chose que ce que je cherchais. En mars 1979, tu écrivais:

Avant de naître je fus mort longtemps. Avaleur de valeurs mais aussi avalé des avalés. Je ne savais pas encore que l'écriture anarchique serait mon laxatif, me ferait chier la Parole ${ }^{3}$.

Voilà bien une intention précise que celle de naître. Lorsqu'on se croit mort mais qu'on refuse de mourir, il se peut bien que toute notre énergie soit axée sur cette idée de survivance. S'il faut pour cela faire éclater tout ce qui nous entoure, nous le ferons, il ne faut pas en douter. «Mieux vaut la fin du monde qu'une égratignure à mon petit doigt», disait un philosophe. Et Whitehead ajoute:

3. Roger Savoie, “Phagie. Petit traité de cannibalisme”, Nouvelle Barre du jour, $\mathrm{n}^{\circ}, 76,1979, \mathrm{p}$ 58 . 
Plus d'un homme de science a patiemment inventé des expériences dans l'intention de soutenir sa croyance que les opérations animales ne sont motivées par aucune intention. Il a peut-être employé ses loisirs à écrire des articles pour prouver que les êtres humains sont comme les autres animaux, de sorte que l'intention est une catégorie sans pertinence pour expliquer leurs activités corporelles, y compris les siennes propres. Les hommes de science animés par I'intention de prouver qu'ils sont dépourvus d'intention constituent un sujet d'étude intéressant ${ }^{4}$.

En quoi le fait d'avoir une intention, si peu précise qu'elle soit, peut-elle enlever de la valeur à un travail d'écriture anarchique? Je me le demande justement. Pourquoi ce mépris face au but? Est-ce du mépris? Il faut, je crois, avoir cru énormément pour vouloir tant en finir avec les croyances. L'indifférence n'est réelle que lorsqu'on ne sent plus le goût d'écrire, de penser, de déchiqueter les choses et les êtres. Lorsqu'une chose ou un être ne fait plus «sens", je n'en parle plus, je ne tiens même pas à réfléchir sur cette chose ou cet être. Quand Sartre dit: «Si Dieu existe, ça ne m'intéresse pas», il affiche clairement ses intentions, il formule devant tous un désir précis de ne pas se préoccuper d'un sujet qui fait sens pour d'autres mais pas pour lui. Ainsi, l'insistance à dire qu'il ne faut plus croire a fini par me faire douter de la validité de tes propositions. «Mon livre, diras-tu, ne contient aucune proposition, et s'il s'en trouve c'est le hasard qui les a mises là.» D'accord, parfaitement d'accord, mais elles ne sont pas pour autant inexistantes ou irréelles. J'admets volontiers que leur apparition est surprenante, mais je suis un lecteur, alors je me permets simplement de lire ce que tu t'es contenté d'écrire. À ce titre, ton livre ne t'appartient plus, et ce serait du terrorisme que de désirer guider ma lecture. Pas plus que toi, je n'ai le goût de m'en faire imposer!

Revenons maintenant au désir, ce magma dans mon corps, ce corps qui en est tout entier rempli. Le désir, ce rien et ce tout, ce fragment et cette totalité. Le désir est là, incontrôlable et incontrôlé. D'où la difficulté d'en parler, d'où l'impossibilité d'en dire la "vérité», d'où l'incompréhension qui me monte à la tête. Dualisme ancien, mais vivace que celui de la tête et du corps. Dualisme qui a fini par s'inscrire en moi comme une certitude et

4. A.N. Whitehead, La fonction de la raison, Payot, Paris, 1969, p. 111-112. 
qui m'empêche de vivre tous mes «moi», qui prétend m'organiser en «un» alors que je suis «multitude».

Le désir est fragmenteur. Il diffracte, il démultiplie, il éparpille ( . . .). L'homme de désir dit: tu n'es rien, tu deviens, tu parais une multitude de visages à la fois, tu n'es pas ici ou là, tu es aussi ailleurs et nulle part. Ce qu'un corps désirant produit échappe à son contrôle, il ne peut pas en être entièrement responsable même s'il sait qu'il en est le déclencheur ${ }^{5}$.

Il m'arrive parfois de choisir un mot parmi d'autres, un concept ou même un acte précis. De me demander, par exemple, pourquoi cet homme a tué cette femme. Et je questionne tout ce qui peut être questionné. De la plus ridicule question à l'interrogation philosophique la plus sérieuse. Si je reconstruis l'histoire de cet homme, je me rends vite compte que des milliers de raisons viennent s'empiler pour justifier son acte. Ces raisons, quoique insatisfaisantes, finissent pas donner du «sens». Mille autres raisons viendront appuyer aussi la thèse contraire. Son acte est explicable et injustifiable. Toute la justice et toutes les théories explicatives reposent sur l'arbitraire, car il m'est impossible de déterminer l'intention précise de cet homme. Lui-même serait probablement incapable de déterminer exactement ce pourquoi il a tué. Quelque chose à un moment ou l'autre a été tué en lui, quelque chose comme du «sens». Peut-être tient-il alors cette femme responsable de cet assassinat, et c'est pour cette raison (et d'autres dont elle n'est qu'en partie responsable et même pas du tout) qu'il va soudainement focaliser en elle tout son mal. Mais je le répète, toute cette histoire demeure arbitraire. Les règles, toutes les règles sont arbitraires, et pourtant quelque part quelqu'un devrait être en mesure de posséder une autorité pour trancher ce qui à première vue ne se tranche pas. Quelque part quelqu'un devra procéder sur cette affaire, à tout le moins pour éviter que l'acte se répète à l'infini. Ce «quelqu'un» sera inévitablement répressif et oppresseur. "La liberté elle-même contient son propre réservoir d'esclavage ${ }^{6}$ », et personne ne viendra contester une telle affirmation. Mais le débat que tu soulèves, Rousseau l'a déjà exposé des milliers de fois. C'est pourquoi le désir, même dans la définition que tu en donnes, même lorsqu'il fragmente et

5. Le philosophe chat, p. 106.

6. Ibid., p. 102. 
que son contrôle est en partie impossible, le désir, donc, change. Je ne dis pas encore qu'il évolue, il change, il devient autre chose, il passe comme la vie et le temps.

Lorsque Rousseau prépara sa réponse à ceux qui l'accusaient de travailler à l'élaboration d'un gouvernement du peuple (ce qui constituait pour eux une mise en question inadmissible de l'autorité), il savait qu'il était perdu d'avance et qu'il ne serait pas entendu. Ses ennemis les plus féroces lui disaient que «tout est bien et qu'ainsi la Providence est justifiée», et lui répliquait:

... si tout est bien comme il est, tout était bien comme il était avant qu'il y eût des Gouvernements et des lois; il fut donc au moins superflu de les établir (. . .). Laissez aller tout comme il pourra, afin que tout aille toujours bien. Si tout est le mieux qu'il peut être, vous devez blâmer toute action quelconque; car toute action produit nécessairement quelque changement dans l'état où sont les choses au moment qu'elle se fait. On ne peut donc toucher à rien sans mal faire; et le quiétisme le plus parfait est la seule vertu qui reste à l'homme? .

C'est dans ce "quiétisme» que dénonce Rousseau, celui-là même qui préconisait ailleurs un retour à l'homme sauvage, que semble baigner ton désir. J'ai toujours pensé que c'est un peu trop lui en donner que de le laisser vivre sans une certaine forme d'esclavage! Bien sûr, mon désir se branche à une autre machine. Bien sûr, il lui arrive de mal se brancher, je sais aussi que c'est là une forme d'apprentissage, et je m'en voudrais de lui refuser le droit d'apprendre simplement parce qu'il est ou doit être anarchique. Je veux qu'il me guide, mais je veux parfois le guider aussi, car toutes les connections, je le sais trop bien, sont possibles.

Le corps n'est pas ravalé au rang de la valeur, le corps est criblé de prises de courants, d'interrupteurs, d'émetteurs, de récepteurs, il fait passer de l'énergie, il en capte, il en produit, il en reçoit, il en coupe . $^{8}$

Pourrai-je un jour éviter les connections qui me font mal? Pourrai-je éviter les connections qui blessent l'autre? Ça aussi c'est du désir. Je ne sais pas toujours ce que c'est que faire le bien. Je n'ai pas toujours le désir de faire le bien, mais il m'arrive d'être

\footnotetext{
7. J.-J. Rousseau, «Lettre à M. Philopolis», in Écrits politiques, 10/18, $\mathrm{n}^{\circ} 726$, Paris, 1972, p. 21-22.

8. Le philosophe chat, p. 54.
} 
bon, il m'arrive aussi qu'on me l'apprenne comme tout le reste. «Les prises de courants» dont tu me parles, elles enregistrent aussi. Mon corps est mémoire, sans cela il y a longtemps que j'en aurais fini avec le désir et peut-être avec la vie. Je joue sur tous les tableaux électriques à la fois, mais je finis par prendre de l'expérience, par me ménager, par désirer ne pas me répéter, par ménager l'autre aussi à l'occasion. Ce n'est pas en soi une question de stabilité à rechercher, c'est bien plus des formulations pratiques et théoriques à retenir pour mieux vivre.

Tu me diras encore une fois que l'intention est douteuse dans tout cela, qu'au niveau du corps et du désir ce genre de décision est impossible. Moi, je crois peut-être encore plus follement que toi, peut-être encore plus irrationnellement que tout est possible, même dans l'ordre du corps et du désir. Que ma raison n'est pas toujours une barrière au corps et au désir, qu'elle me permet un contrôle que j'apprécie, et qu'elle me guide. Les philosophes ont longtemps voulu être des guides pour les autres; il se sont cantonnés sur le terrain de l'éthique, croyant ainsi théoriquement pouvoir offrir aux autres des règles de conduite. Je sais combien nous en sommes gênés aujourd'hui. Nous voudrions bien effacer ces périodes de l'histoire de la philosophie où nous avons couché avec le pouvoir chaque fois que nous en avons eu l'occasion. Il y a des raisons au fait que nos objets de réflexion sont soudainement devenus la folie, l'anarchie, l'anti-pouvoir, l'anti-violence, l'antiguerre. Nous avons été de ceux qui savions fournir aux dirigeants les raisons pour tuer, violer, ravager, détruire et torturer. Pas plus que ceux des autres, nos idéaux ne furent d'une pureté exemplaire. C'est très encombrant lorsqu'on se veut des maîtres de vie et de sagesse.

Nous devrions savoir que l'histoire ne s'efface pas, que nous la traînons avec nous, que nous sommes même, chaque jour et chaque nuit, individuellement, un fragment d'histoire et que tôt ou tard nous rendrons aux autres des comptes. Lorsque tu écris:

Vingt-cinq années d'humanisation intensive, il y en avait des ordres à anarchiser ou plutôt un désordre toujours là à faire parler. Croyez-moi j'en avais des suicides à perpétrer, de l'humain à transsuder, de l'anthropos à déculotter ${ }^{9}$.

9. NBJ, ibid., p. 61. 
Puis-je te dire, le plus simplement du monde, que je te crois. Mais alors il te faut admettre avec moi que Le philosophe chat a une histoire, un but, un ordre et un désordre. À mon avis, toute écriture théorique (peu importent ses prétentions) devrait en un sens dévoiler du même coup l'autobiographie de son auteur. Quelque chose nous arrive qui provoque en nous le désir de dire les choses d'une quelconque façon. "Aucune théorie ne peut se développer sans rencontrer une espèce de mur, et il faut la pratique pour percer le mur ${ }^{10}$ ", dit Gilles Deleuze. Les philosophes se réfugient fréquemment dans l'ordre théorique pour dire ce qui leur fait mal. Ainsi, la théorie ne serait rien d'autre que manière de se raconter. D'où, peut-être, l'immense jeu de séduction qui s'y joue et qui se joue parfois des autres.

Le philosophe chat est un livre séduisant, attirant, on s'y glisse en boule, il est chaud. Mais la séduction est un jeu, elle est aussi un piège. Toujours quelque part des intentions se cachent, des messages se camouflent. Attention! je ne veux pas dire qu'il n'y aurait que de la séduction dans ton livre, mais les «ruses» de l'anti-philosophe ne sont pas toutes claires. Le philosophe chat n'est pas une véritable mise à nu; il semble bien qu'une telle entreprise soit de toute manière vouée à l'échec. Mais une bataille est menée contre un «autre» en toi. Un, parmi la multitude en toi, parmi les centaines qui t'habitent, qui se nomme «le croyant». Il a dû, je le répète, être bien fort, bien présent, bien envahissant pour que tu le pourchasses ainsi dans tous les recoins possibles. Toutes nos croyances, même les plus belles, même et surtout les plus certaines, subissent une ou des dégradations. Nous sommes d'un siècle qui au premier coup d'oeil ne croit plus guère. Tant et si bien que tout est maintenant possible.

On s'arrange avec ce que les générations précédentes nous ont légué, mais on ne peut s'empêcher de désirer refaire le monde un jour ou l'autre, du moins contribuer si possible à quelques petites transformations afin d'éviter d'être une sombre réplique du passé, mais aussi pour produire notre propre passé.

Je sais bien que l'idée de ne plus croire en rien est séduisante. Je sais bien qu'il s'agit là d'un geste sain lorsque l'on ne cesse de se briser et de s'effondrer face à l'athéisme des autres et au nôtre. Je

10. L'Arc, $n^{\circ} 49$, entretien Foucault/Deleuze, p. 3. 
sais bien qu'on finit par perdre le goût de parler, d'écrire, de dire. Qu'on a soudainement une frousse épouvantable d'être de nouveau trompé. On peut même imaginer que c'est finalement notre propre manière d'être qui produit de telles manifestations d'incroyance autour de soi. L'expérience répétée de la fin des croyances me convainc de ne plus croire en rien, de me laisser guider par le désir, d'être mon corps et rien d'autre.

Le désir c'est un non-lieu, où rien signifie tout, lieu du paradoxe, de l'impolitesse. Sa révolution est polissonne et délinquante ${ }^{11}$.

Des attributs théistes sont constamment utilisés pour parler du désir. Chacun de ces attributs possède son lot de séduction. Toutes les révolutions se sont posées comme polissonnes et délinquantes, et où en sommes-nous maintenant? Cette recherche d'un désordre en moi, pour combattre l'ordre qu'on y a mis, suppose encore une fois la réinvention du débat nature/culture. Mais tout s'épuise et nos révolutions d'aujourd'hui ne seront que l'arrièregarde de demain. Nous n'en finissons pas de nous faire, de nous refaire, de nous défaire.

Si vous vous engagez dans une aventure amoureuse, sans tenue d'apparat, sans trop de retenue, sans que ce soit la poursuite d'une idée d'amour, vous ne savez pas où ça va vous conduire, vous vous mettez dans un état d'incertitude, vous brouillez toutes les pistes et vous faites sans le vouloir des atteintes à la moralité. Vous déclinez toute responsabilité car vous ne pouvez pas répondre de ce qui vous arrivera $^{12}$.

Je finirai toutefois par poser des actes. Je finirai bien par m'engager quelque part, je finirai bien par avoir une idée de l'endroit ou ça va. Et si je le sais, il faudra bien que j'en parle, que je l'écrive, que je partage cette «aventure amoureuse». Si l'autre ne vas pas à la même place, ou ne veut tout simplement pas y aller, ou me renvoie à mes incertitudes premières, ne devrais-je pas être en mesure d'établir avec l'autre des règles minimales de moralité?

Il faut bien le dire, même dans une aventure amoureuse où l'on ne se demande rien, où l'on ne poursuit pas une idée d'amour, il y a au moins une règle minimale: ON NE SE DEMANDE

11. Le philosopbe chat, p. 58 .

12. lbid., p. 92. 
RIEN. Même pas du plaisir, même pas de la tristesse, même pas un acte. ON NE SE DEMANDE RIEN. Alors, qu'est-ce qu'on risque? Que deux désirs se croisent, au-delà du conscient et que ça jouisse malgré tout. Il se peut bien que ce genre d'expérience soit vécu par nous tous, il se peut bien que nous l'ayons souhaité plus ou moins (ce qui cache alors des intentions). Une sorte de DERNIER TANGO À PARIS, où le désir fait son apparition brutalement, mais très explicitement. Je crois qu'à chaque fois nous en évaluons les risques aussi, avant ou après, peu importe. Il en est plusieurs d'entre nous (et les femmes tout particulièrement) qui appellent cela: SE FAIRE PASSER DESSUS. On finit même par se dire qu'à être ainsi vulnérable, le risque est encore trop grand. Chaque fois que le désir brut vient, chaque fois que je veux lui dire oui, je risque. Notre société a inventé une nouvelle exploitation, celle du désir, c'est séduisant, et ils sont nombreux les «magnats» du désir, les théoriciens de l'irresponsabilité de nos actes.

Je terminerai justement sur ce qui m'apparaît être à la veille de devenir le nouveau mythe de la décennie: le désir incontrôlé et incontrôlable. Le désir défini comme une sorte de mors aux dents du corps est, chez bien des mâles "raisonnables", une manière de s'épancher. Il suffit alors d'écrire un livre sur le désir, d'en chanter les bienfaits et surtout de montrer que le désir est strictement FÉMININ. Croyant ainsi découvrir un nouveau monde (ce qui constitue une caractéristique très masculine), ils tombent tête baissée dans la dichotomie masculin/féminin. Simple invention de nouvelles catégories de séduction pour remplacer les ruses éculées des chasseurs du passé. Division binaire qui place le désir du côté des femmes et la raison du côté des hommes. Malheureusement, je crois que ton texte n'échappe pas à ce modèle:

Or ce qui m'arrivait était tout autre. Par exemple un bizarre processus de féminisation, par d'autres biais que l'homosexualité. Félinité et féminité cö̈ncidaient. La femme, lorsqu'elle jouit, perd littéralement la tête, et pour longtemps, elle ne finit plus de se perdre, le nord, les feuilles, pied, son latin, conscience. Je m'engageais dans une périlleuse entreprise de perdition ${ }^{13}$.

Voilà: pour perdre la tête, devenez femme. Puis-je douter? Puis-je y voir une solution de facilité, une généralisation hâtive, 13. Ibid., p. 164. 
un gonflement du plaisir féminin, un nouveau mythe? Non pas qu'il faille demeurer viril, fort, sans larmes et raisonnable, ce n'est pas du tout ce que j'avance. Mais le désir n'est pas en soi féminin ou masculin. Le désir demeure flou, autant là qu'ailleurs. Ce n'est pas parce qu'un homme jouit qu'il devient pour autant femme.

Mais ce qui m'apparaît plus grave encore, c'est que les dernières pages de ton livre fixent des catégorisations que tu avais justement su très bien éviter jusque là. Peut-être que cette dernière partie qui porte comme sous-titre: «comme une autobiographie» vient vraiment trop tard. Que veut dire par exemple la phrase suivante:

La philosophie est mâle, on le dit. Elle est plutôt neutre et asexuée. Les femmes philosophes sont toujours mauvaises: c'est là un témoignage d'admiration, non une injure. Qu'elles se mettent à philosopher (sans trop singer le discours viril) et on verra la saignée de la philosophie, mais ce ne sera pas du sang qui en sortira, seulement de l'air ${ }^{14}$.

Qu'est-ce à dire? Qu'il y aurait «un discours viril» qui demeure «neutre et asexué»? Encore une fois, je doute. De plus, cette recommandation faite aux femmes-philosophes de «ne pas singer» ce discours m'apparaît elle aussi très normative. $\mathrm{Y} a-t$-il une femme quelque part pour dire aux hommes de cesser de «singer» le «discours (et même la jouissance ... féminin», d'en faire le lieu même du «désir»? Toute cette histoire de masculin/féminin demeure l'élément le plus confus de ces dix dernières années. À lire les nouvelles propositions qui nous proviennent d'un peu partout, on a souvent l'impression qu'hommes et femmes s'inventent tout simplement de nouveaux discours de séduction sans que pour autant des transformations majeures se produisent. Ils pullulent actuellement les hommes qui ont découvert qu'il faut être un tant soit peu féministe pour séduire les femmes. De l'autre côté, il y a des femmes qui s'efforcent de prouver aux hommes qu'elles sont capables de vivre comme nous. Mais pendant ce temps, nous souffrons tous plus ou moins de ces masques que nous ajoutons sur les autres masques traditionnels. Nos tristesses et nos joies sont ailleurs et nous oublions très souvent de les vivre et surtout de les partager.

14. Ibid., p. 165 . 
Je sais bien que cette découverte de la "féminisation" pour un philosophe peut être très importante. Je sais bien que les hommes et les femmes sont des fabrications sociales et qu'il faut bien du temps et de la patience pour découvrir qu'il y a là comme une montagne de mensonges.

Le philosophe chat demeure un beau livre. Il ne voulait pas être beau, il l'est devenu. Je doute pourtant qu'il soit un véritable traité d'anti-philosophie. Parler du désir et du corps n'est pas en soi une matière, un sujet anti-philosophique. Le désir et le corps sont partout. Un peu dans tout, dans la raison comme dans la volupté, dans la jouissance comme dans la souffrance, dans l'écriture et dans la main qui effleure un corps. Il n'est pas nécessairement femme, il n'est pas nécessairement homme. Il est.

Une dernière question. En 1978, on annonçait, dans le collectif Sortir des Éditions de l'Aurore, un livre à paraître de Roger Savoie: Le philosophe chat ou mal-traité d'anti-philosophie. En 1980, paraît: Le philosophe chat ou les ruses du désir. Est-ce une «ruse» de l'éditeur ou de l'auteur, ce changement du titre? Car je pense que le mot «désir» fait vendre un peu plus que le mot "philosophie», même si on le fait précéder d'un «anti»! Commerce oblige, je suppose. Que faut-il y voir?

Département de philosophie Cegep François-Xavier Garneau Juillet 1980 